\title{
An Unusual Case of Uterovesical Polyp in a Patient Following Repeated Caesarean Section
}

\author{
Okwudili C. Amu, Emmanuel A. Affusim, Ugochukwu U. Nnadozie, and Balantine U. Eze
}

\section{ABSTRACT}

$\mathrm{NE}$ is a 36yr old petty trader who has had four previous caesarean sections. She went for her routine abdominal ultrasonography at 36weeks of her fifth pregnancy and an incidental posterior bladder polypoid mass was found with a normal singleton fetus. Patient declined cystoscopy but accepted exploration of bladder at same time with a caesarean section. At Surgery, a polyp was found arising from the lower uterine segment traversing the thin posterior bladder wall into the bladder. The polyp was carefully dissected out and separated from bladder. Bladder and uterus were repaired, and patient had uneventful recovery.

Keywords: Bladder exploration, repeated caesarean section, uterovesical polyp, Youssef syndrome.

\section{INTRODUCTION}

Repeated caesarean sections in a woman carries the risk of adverse maternal outcomes. Complications arising from this includes dense intraperitoneal adhesions and fivefold increase in premature deliveries [1]. Repeated caesarean sections are occasionally associated with abnormalities of the placenta which may cause excessive and dangerous bleeding in late pregnancy and at the time of delivery [2]. There has been reports of cyclical menouria from uterovesical fistulas following caesarean sections [3]. Bladder endometriosis arising as a complication of caesarean section has also been documented [4].

However, uterovesical polyp probably arising as a complication of multiple caesarean section was not found in literature. We present an unusual case of uterovesical polyp in a woman after undergoing repeated caesarean sections.

\section{CASE REPORT}

Mrs NE is a 36 years old petty trader, who presented to the hospital in her fifth pregnancy after previous four caesarean sections. She has three alive children, two girls and a boy.

She underwent a routine ultrasonography at 36weeks
Submitted : September 8, 2021

Published. : November 13, 2021

ISSN: 2593-8339

DOI: $10.24018 /$ ejmed.2021.3.6.1049

O. C. Amu*

College of Medicine, University of Nigeria, Enugu Campus, Nigeria.

(e-mail: okwudiliamu@gmail.com)

E. A. Affusim

College of Medicine, Odumegwu

Ojukwu University, Awka, Anambra

State, Nigeria.

(e-mail: primusal@gmail.com)

U. U. Nnadozie

Federal University Teaching Hospital,

Abakiliki, Ebonyi State, Nigeria.

(e-mail: ugodozie1@gmail.com)

B. U. Eze

Department of Surgery, Enugu State

University of Science and Technology,

Enugu, Nigeria.

(e-mail: balantine.eze@esut.edu.ng)

*Corresponding Author gestational age, and the ultrasound revealed a normal singleton fetus in cephalic presentation. However, it also showed a posterior bladder polypoid mass. She was completely asymptomatic: no observed urinary symptoms nor gross haematuria.

Previous surgical history revealed she had done four caesarean sections. The first two by a general practitioner and the last two by a gynaecologist. The gynaecologist noted in the third caesarean section that the bladder was high riding with its thin posterior wall plastered to the lower uterine segment with adhesions

Abdominal examination revealed a midline sub-umbilical abdominal scar and a 38weeks gravid uterus. Urinalysis and microscopy showed no microscopic haematuria. A repeat ultrasound by a consultant radiologist revealed same features as the previous one done by a resident. Patient was booked for a cystoscopy, but she declined due to financial constraints as she had already saved money only for an elective caesarean section (she did not have health insurance).

A decision was taken with the gynaecologist to explore the bladder at the time of the elective caesarean section, and she accepted. She and her spouse were also counselled and accepted bilateral tubal ligation.

She was admitted on the 4th of September 2014, evaluated 
for fitness for surgery and two units of compatible blood were grouped and cross-matched. Operative technique and findings: under spinal anaesthesia and in supine position with urethral catheter insitu, a lower midline abdominal incision excising the previous scar was used to gain access to the peritoneal cavity. Adhesions involving the gut and uterus were all released, and incision made on uterine lower segment to deliver a live male fetus weighing $3.8 \mathrm{~kg}$. Placenta was delivered and uterine ceasearean incision was closed in two layers with vicryl size 2 . Bilateral tubal ligations were done.

Abdominal packs were employed to retract the uterus and gut upwards and a cystotomy was made which revealed a haemorrhagic polypoid bladder mass at the posterior wall of the bladder (Fig. 1, 2 and 3). Further attempts to mobilize the bladder revealed a thin posterior wall of the bladder plastered intimately to the lower uterine segment.

The polyp was found to be arising from the lower uterine segment traversing the thin posterior bladder wall into the bladder. The polyp was carefully dissected out and separated from the bladder. The resulting defect in the bladder was carefully repaired transversely in two layers using vicryl size 1. The polyp was dissected off the uterus and found to be communicating with the uterine cavity. The uterine defect left after excision of the polyp was closed in two layers with chromic size 2.

Postoperatively, patient was commenced on oral feeding after $48 \mathrm{~h}$, drain was removed after 5 days and urethral catheter removed after 2 weeks. She did well and was discharged.

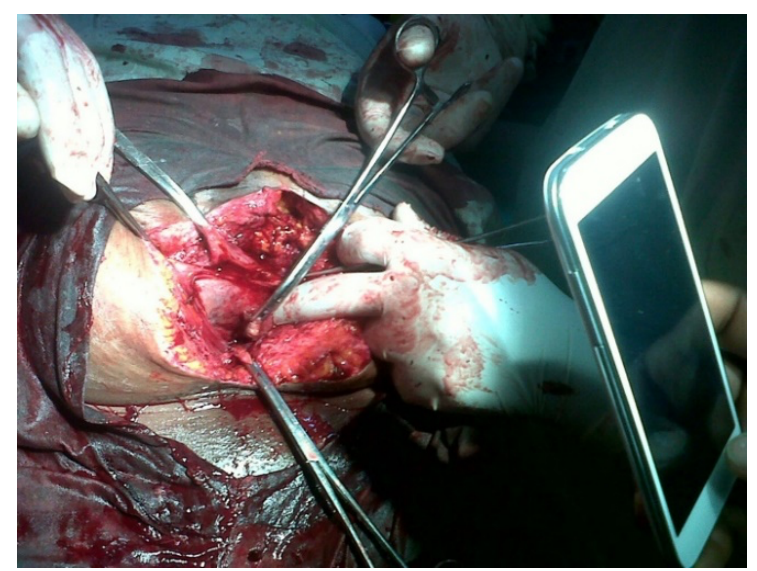

Fig. 1. Polyp at posterior wall of bladder.

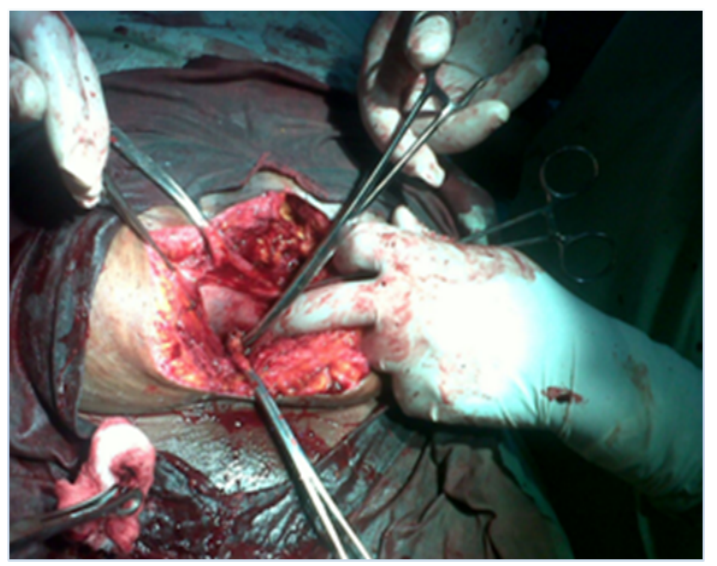

Fig. 2. Bladder polyp.

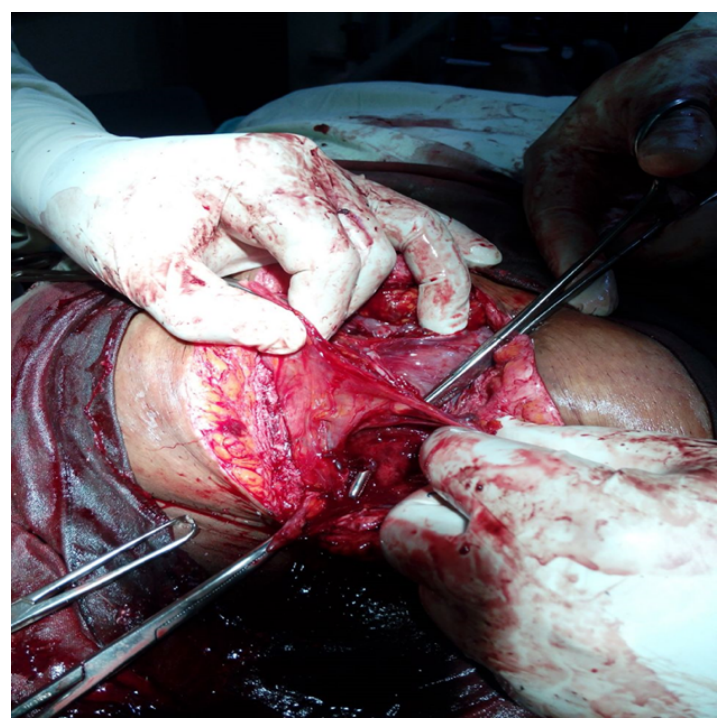

Fig. 3. Haemorrhagic bladder polyp.

\section{A. Histology Report}

Sections show dilated and congested blood vessels of varying sizes in a background of decidualised cells and siderophages. There are ghost outlines of glandular structures. There is no atypical proliferation present. Diagnosis- Iatrogenic endometrial vesical explants.

\section{DISCUSSION}

Maternal complications arising from repeated caesarean sections are well known however the phenomenon of a polypoid mass arising from the uterus into the bladder has not been mentioned in the literature.

This patient was completely asymptomatic however the decision to operate is based on the fact that in the long term the polypoid mass could rupture, and a fistulous tract could be established leading to cyclical menouria- Youssef syndrome [5]. Also, the psychological trauma from knowledge of having an unresolved pathology in the bladder could be daunting.

In retrospect, a cystoscopy, and a likely decision to do a trans-urethral resection of bladder tumor in this patient may not have solved this patient's problem as it would have simply established a fistula since that procedure would not handle the uterine component. Urologist certainly should think twice before resecting a polypoid mass arising from a posterior bladder wall in any woman following repeated caesarean section.

The histology report confirmed an iatrogenic endometrial vesical explant. A possible cause is inadvertent injury to the bladder wall while repairing the uterus since the gynaecologist noted at the third caesarean section that the posterior bladder wall was thin and plastered to the lower segment of the uterus.

This injury may have been in form of a bite by the suturing needle incorporating the posterior bladder wall during the repair of the lower uterine segment and subsequent devitalization of the already thin bladder wall with sloughing off and apparent ingrowth of endometrial tissue even though one would have expected just a fistula. Possible ways to avert this problem could have been proper identification of the bladder wall and bluntly retracting it away from the lower 
uterine segment after initial urethral catheterization for bladder decompression.

In conclusion, repeated caesarean sections are fraught with adverse maternal outcomes including uterovesical polyp. Experienced gynaecologists should handle these cases of repeated caesarean sections to reduce possibility of these complications.

\section{REFERENCES}

[1] U. Dilek, G. Ozlem, K. Sefa, O. Arzu, U. Mustafa, and M. Tamer, "Muitiple repeat caesarean section: Is it safe?," European journal of obst. \& Gynae and reproductive biology, vol. 19, pp. 171-175, 2005.

[2] B. A. Gedik, A. Akyol, B. Bingol, D. Cakmak, A. Sargin, R. Uncu, and Y. Ceylan, "Multiple repeated caesarean deliveries: operative complications in the fourth and fifth surgeries in urgent and elective cases," Taiwan J Obstet Gynecol, vol. 49, pp. 425-431, 2010.

[3] J. T. Mutihir, O. Amu, and H. Liman, "Successful repair of iatrogenic cyclical menouria: A case report," Nigerian journal of clinical practice, vol. 13, pp. 344-346, 2010.

[4] G. N. Cruz, Q. A. Linares, R. C. Cuesta, R. L. Clemente, and C. A. Castaneda, "Bladder endometriosis in a patient with caesarean history," Arch Esp. Urol, vol. 55, pp. 194-197, 2002.

[5] A. D. Youssef, "Menouria following lower segment caesarean section: A syndrome," American Journal of Obstetrics and Gynaecology. vol. 73, pp. 759-767, 1957. 